PROCEEDINGS OF THE

AMERICAN MATHEMATICAL SOCIETY

Volume 131, Number 2, Pages 647-654

S 0002-9939(02)06717-5

Article electronically published on June 27, 2002

\title{
A WEAKLY STEGALL SPACE THAT IS NOT A STEGALL SPACE
}

\author{
WARREN B. MOORS AND SIVAJAH SOMASUNDARAM
}

(Communicated by Jonathan M. Borwein)

\begin{abstract}
A topological space $X$ is said to belong to the class of Stegall (weakly Stegall) spaces if for every Baire (complete metric) space $B$ and minimal usco $\varphi: B \rightarrow 2^{X}, \varphi$ is single-valued at some point of $B$. In this paper we show that under some additional set-theoretic assumptions that are equiconsistent with the existence of a measurable cardinal there is a Banach space $X$ whose dual, equipped with the weak* topology, is in the class of weakly Stegall spaces but not in the class of Stegall spaces. This paper also contains an example of a compact space $K$ such that $K$ belongs to the class of weakly Stegall spaces but $\left(C(K)^{*}\right.$, weak $\left.{ }^{*}\right)$ does not.
\end{abstract}

\section{INTRODUCTION}

We say that a Banach space $X$ is a weak Asplund (almost weak Asplund) [Gâteaux differentiability] space if every continuous convex function defined on a non-empty open convex subset $A$ of $X$ is Gâteaux differentiable at the points of a residual (everywhere second category)[dense] subset of $A$. While it is easy to see that every weak Asplund space is almost weak Asplund and every almost weak Asplund space is a Gâteaux differentiability space, the status of the reverse implications remains unresolved. In the study of weak Asplund spaces C. Stegall introduced the following class of topological spaces which are defined in terms of minimal uscos. Recall that a set-valued mapping $\varphi: X \rightarrow 2^{Y}$ acting between topological spaces $X$ and $Y$ is called an usco mapping if for each $x \in X, \varphi(x)$ is a non-empty compact subset of $Y$ and for each open $W$ in $Y,\{x \in X: \varphi(x) \subseteq W\}$ is open in $X$. An usco mapping $\varphi: X \rightarrow 2^{Y}$ is called a minimal usco if its graph does not properly contain the graph of any other usco defined on $X$ and a topological space $Y$ is said to belong to the class of Stegall spaces if for every Baire space $B$ and minimal usco $\varphi: B \rightarrow 2^{Y}, \varphi$ is single-valued at some point of $B$ or equivalently, $\varphi$ is single-valued at the points of a residual subset of $B$, (see, Theorem 3.2.6 of [1]). Here we study the class of weakly Stegall spaces which was first introduced in [2]. We say that a topological space $Y$ belongs to the class of weakly Stegall spaces if for every complete metric space $M$ and minimal usco $\varphi: M \rightarrow 2^{Y}, \varphi$ is single-valued at some point of $M$. The study of this class of topological spaces is very natural, especially in the setting of Banach spaces. Since for a Banach space $X$ if $\left(X^{*}\right.$, weak $\left.{ }^{*}\right)$ is a weakly Stegall space, then

Received by the editors September 12, 2001.

2000 Mathematics Subject Classification. Primary 54C60, 26E25, 54C10.

Key words and phrases. Weak Asplund, almost weak Asplund, Stegall space, weakly Stegall space.

(C)2002 American Mathematical Society 
$X$ is a Gâteaux differentiability space (but not necessarily a weak Asplund space). This is particularly significant as it has been a long standing open question as to whether every Gâteaux differentiability space is a weak Asplund space. Thus one would ideally like to construct a non-weak Asplund space $X$ such that $\left(X^{*}\right.$, weak*) is a weakly Stegall space. Unfortunately, the construction of such a Banach space remains elusive. However, in this paper we construct a Banach space $X$ such that $\left(X^{*}\right.$, weak $\left.{ }^{*}\right)$ belongs to the class of weakly Stegall spaces but not to the class of Stegall spaces.

The remainder of this paper goes as follows. In Section 2 we introduce a topological game that is related to the class of weakly Stegall spaces. Then we use this game to characterize those spaces belonging to the class of weakly Stegall spaces. We end Section 2 by applying this characterization to a recent example given in [4] to obtain a Banach space $X$ such that $\left(X^{*}\right.$, weak $\left.{ }^{*}\right)$ is a weakly Stegall space but not a Stegall space. Section 3 is then devoted to constructing a weakly Stegall compact space $K_{A}$ such that $\left(C\left(K_{A}\right),\|\cdot\|_{\infty}\right)$ is not almost weak Asplund.

\section{A weakly Stegall but non-Stegall space}

Let $\mathcal{U}$ be any open cover of a topological space $(X, \tau)$. On $X$ we consider the $\mathcal{G}(\mathcal{U})$-game played between two players $\Sigma$ and $\Omega$. Player $\Sigma$ goes first (always!) and chooses a non-empty subset $A_{1}$ of $X$. Player $\Omega$ must then respond by choosing a non-empty relatively open subset $B_{1}$ of $A_{1}$ of the form: $B_{1}:=B_{1}^{*} \cap A_{1}$ with $B_{1}^{*} \in \mathcal{U}$. Following this, player $\Sigma$ must select another non-empty subset $A_{2} \subseteq B_{1} \subseteq A_{1}$ and in turn player $\Omega$ must again select a non-empty relatively open subset $B_{2}$ of $A_{2}$ of the form: $B_{2}:=B_{2}^{*} \cap A_{2}$ with $B_{2}^{*} \in \mathcal{U}$. Continuing this procedure indefinitely the players $\Sigma$ and $\Omega$ produce a sequence $\left\{\left(A_{n}, B_{n}\right): n \in \mathbb{N}\right\}$ of pairs of nonempty subsets called a play of the $\mathcal{G}(\mathcal{U})$-game. We shall declare that $\Omega$ wins a play $\left\{\left(A_{n}, B_{n}\right): n \in \mathbb{N}\right\}$ if, $\bigcap_{n=1}^{\infty} B_{n}$ is at most one point. Otherwise, the player $\Sigma$ is said to have won the play. By a strategy $t$ for the player $\Sigma$ we mean a "rule" that specifies each move of the player $\Sigma$ in every possible situation. More precisely, a strategy $t:=\left(t_{n}: n \in \mathbb{N}\right)$ for $\Sigma$ is a sequence of set-valued mappings such that $\emptyset \neq t_{n+1}\left(B_{1}, B_{2}, \ldots, B_{n}\right) \subseteq B_{n}$ for all $n \in \mathbb{N}$. The domain of each mapping $t_{n}$ is precisely the set of all finite sequences $\left\{B_{1}, B_{2}, \ldots, B_{n-1}\right\}$ of length $n-1$ with each $B_{j}$ being a non-empty relatively open subset of $t_{j}\left(B_{1}, B_{2}, \ldots, B_{j-1}\right)$ of the form $B_{j}:=B_{j}^{*} \cap t_{j}\left(B_{1}, B_{2}, \ldots, B_{j-1}\right)$ with $B_{j}^{*} \in \mathcal{U}$. (Note: the sequence of length 0 will be denoted by $\emptyset$ ). Such a finite sequence $\left\{B_{1}, B_{2}, \ldots, B_{n-1}\right\}$ (infinite sequence $\left.\left\{B_{n}: n \in \mathbb{N}\right\}\right)$ is called a partial t-play $\left(t\right.$-play). A strategy $t:=\left(t_{n}: n \in \mathbb{N}\right)$ for the player $\Sigma$ is called a winning strategy if each infinite $t$-play is won by $\Sigma$. We will say that the $\mathcal{G}(\mathcal{U})$-game on $X$ is $\Sigma$-unfavourable if the player $\Sigma$ does not have a winning strategy in this game.

We say that a subset $Y$ of a topological space $(X, \tau)$ has countable separation in $X$ if there is a countable family $\left\{O_{n}: n \in \mathbb{N}\right\}$ of open subsets of $X$ such that for every pair $\{x, y\}$ with $y \in Y$ and $x \in X \backslash Y,\{x, y\} \cap O_{n}$ is a singleton for at least one $n \in \mathbb{N}$. For a completely regular topological space $(X, \tau)$ we shall simply say that $X$ has countable separation if in some compactification $b X, X$ has countable separation in $b X$. It is shown in [7] that if $X$ has countable separation in one compactification, then $X$ has countable separation in every compactification and that every Cech-analytic space has countable separation. A set-valued mapping $\varphi: X \rightarrow 2^{Y}$ acting between topological spaces $X$ and $Y$ is said to be minimal if for 
each pair of open subsets $U$ of $X$ and $W$ of $Y$ such that $\varphi(U) \cap W \neq \emptyset$ there exists a non-empty open subset $V \subseteq U$ such that $\varphi(V) \subseteq W$. This definition is modeled on the characterizing property of minimality of usco mappings, i.e., an usco mapping $\varphi: X \rightarrow 2^{Y}$ is a minimal usco if, and only if, $\varphi$ is a "minimal" mapping. The following theorem may be extracted from Theorems 4,5 and 6 of [5].

Theorem 1. For a completely regular topological space $(X, \tau)$ with countable separation the following properties are equivalent:

(i) $(X, \tau)$ is a weakly Stegall space;

(ii) the $\mathcal{G}(\tau)$-game on $X$ is $\Sigma$-unfavourable;

(iii) every minimal non-empty valued mapping $\varphi: M \rightarrow 2^{X}$ acting from a complete metric space $M$ into $X$ is single-valued at some point of $M$;

(iv) every minimal non-empty valued mapping $\varphi: M \rightarrow 2^{X}$ acting from a complete metric space $M$ into $X$ is single-valued at the points of an everywhere second category subset of $M$;

(v) every minimal non-empty valued mapping $\varphi: B \rightarrow 2^{X}$ acting from an $\alpha$ favourable space $B$ (see, 5] for the definition) into $X$ is single-valued at the points of an everywhere second category subset of $B$.

The previous theorem enables us to establish the relationship between weakly Stegall and fragmentable spaces as well as the relationship between weakly Stegall and almost weak Asplund spaces. Recall that a topological space $(X, \tau)$, endowed with a metric $d$, is said to be fragmented by $d$ if for each $\varepsilon>0$ and each non-empty subset $A$ of $X$ there exists a non-empty relatively open subset $B$ of $A$ such that $d$-diam $(B)<\varepsilon$. In such a case the space is said to be fragmentable. Now in [6] the authors show that a space $(X, \tau)$ is fragmentable if, and only if, the player $\Omega$ has a winning strategy in the $\mathcal{G}(\tau)$-game played on $X$. This contrasts with the situation for weakly Stegall spaces which are characterized by the lack of a winning strategy for the player $\Sigma$ in the $\mathcal{G}(\tau)$-game played on $X$. Hence the distinction between being fragmentable and being weakly Stegall is equivalent to the distinction between $\Omega$ having a winning strategy and $\Sigma$ not having a winning strategy. The relationship between a Banach space $X$ being almost weak Asplund and possessing a weakly Stegall dual space can also be clarified here. It follows from Theorem 1 (iv) and the proof of Theorem 3.2.2 in [1] that every Banach space whose dual, equipped with the weak* topology, is weakly Stegall is almost weak Asplund.

Theorem 2. Let $(X, \tau)$ be a completely regular topological space with countable separation and weight $\kappa \geq \aleph_{0}$. Then $(X, \tau)$ is a weakly Stegall space if, and only if, for every complete metric space $M$ of density at most $\kappa$ and minimal usco $\varphi$ : $M \rightarrow 2^{X}, \varphi$ is single-valued at some point of $M$.

Proof. It is immediately clear from the definition that if $(X, \tau)$ belongs to the class of weakly Stegall spaces, then for every complete metric space $M$ of density at most $\kappa$ and minimal usco $\varphi: M \rightarrow 2^{X}$ there is a point at which $\varphi$ is single-valued. So we consider the converse. Let $\mathcal{B}$ be a topological base for $(X, \tau)$ with cardinality $\kappa$ and let $t:=\left(t_{n}: n \in \mathbb{N}\right)$ be a strategy for the player $\Sigma$ in the $\mathcal{G}(\tau)$-game played on $X$. We need to show that $t$ is not a winning strategy, that is, we need to construct a $t$-play, $\left\{B_{n}: n \in \mathbb{N}\right\}$ in which $\Omega$ wins. To accomplish this we consider a new game on $X$. Namely, we consider the $\mathcal{G}\left(\mathcal{B}^{*}\right)$-game on $X$, where $\mathcal{B}^{*}:=\mathcal{B} \cup\{X\}$. For this game we inductively define a strategy $t^{\prime}:=\left(t_{n}^{\prime}: n \in \mathbb{N}\right)$ for the player $\Sigma$ in terms of the strategy $t$. 
First we define $t_{1}^{\prime}(\emptyset):=t_{1}(\emptyset)$ and then under the assumption that for each $1 \leq j \leq n, t_{j}^{\prime}$ has been defined in such a way that each partial $t^{\prime}$-play of length at most $n$ is a partial $t$-play, we define $t_{n+1}^{\prime}\left(B_{1}, B_{2}, \ldots, B_{n}\right):=t_{n+1}\left(B_{1}, B_{2}, \ldots, B_{n}\right)$. This completes the definition of $t^{\prime}:=\left(t_{n}^{\prime}: n \in \mathbb{N}\right)$. With this definition we see that every $t^{\prime}$-play is a $t$-play. In fact, $t^{\prime}$ is essentially the restriction of the strategy $t$ to the $\mathcal{G}\left(\mathcal{B}^{*}\right)$-game. Next we let $P$ denote the space of all $t^{\prime}$-plays endowed with the Baire metric $d$, that is, if $p:=\left\{B_{n}: n \in \mathbb{N}\right\}$ and $p^{\prime}:=\left\{B_{n}^{\prime}: n \in \mathbb{N}\right\}$, then $d\left(p, p^{\prime}\right)=0$ if $p=p^{\prime}$ and otherwise, $d\left(p, p^{\prime}\right)=1 / n$, where $n:=\min \left\{i \in \mathbb{N}: B_{i} \neq B_{i}^{\prime}\right\}$. It is straightforward to verify that $(P, d)$ is a complete metric space. We claim however that the density of $(P, d)$ is at most $\kappa$. To see this, we first observe that there are at most $\kappa$ partial $t^{\prime}$-plays and that for every partial $t^{\prime}$-play $\left\{B_{j}: 1 \leq j \leq n\right\}$ we may fix a unique extension to a full $t^{\prime}$-play, namely, that defined $B_{k}:=t_{k}\left(B_{1}, B_{2}, \ldots, B_{k-1}\right)$ (i.e., $\left.B_{k}^{*}:=X\right)$ for all $k>n$. One may now check that the set of all these extensions is dense in $(P, d)$. Therefore, the density of $(P, d)$ is at most $\kappa$.

Next we define, as in Theorem 2 of [5], a set-valued mapping $F: P \rightarrow 2^{X}$ by

$$
F(p):=\bigcap_{n=1}^{\infty} B_{n}, \quad \text { where } p:=\left\{B_{n}: n \in \mathbb{N}\right\} .
$$

If $F(p)=\emptyset$ for some $t^{\prime}$-play $p$, then $\Omega$ wins the play and so $t$ is not a winning strategy for the player $\Sigma$ in the $\mathcal{G}(\tau)$-game played on $X$; which completes the proof. Hence we shall assume that $F$ has non-empty values. It now follows, as in Corollary 3 of [5], that $F$ is a minimal mapping. Moreover, by Theorem 6 of [5] there is a dense $G_{\delta}$ subset $G$ of $P$ and a minimal usco $\tilde{F}: G \rightarrow 2^{X}$ such that $F(p) \subseteq \tilde{F}(p)$ for all $p \in G$. Now since $G$ is completely metrizable and has density at most $\kappa$, there exists some $t^{\prime}$-play $p$ in $G$ such that $\tilde{F}(p)$ is a singleton, from which it follows that $F(p)$ is a singleton and thus $p$ is a $t$-play in which $\Omega$ wins. Therefore $t$ is not a winning strategy for the player $\Sigma$ in the $\mathcal{G}(\tau)$-game played on $X$. This completes the proof.

Proposition 1 ([2, Proposition W2, part(b)]). Let $(X, \tau)$ be a topological space and let $\left\{X_{n}: n \in \mathbb{N}\right\}$ be closed subsets of $X$. If each $X_{n}$ is a weakly Stegall space and $X=\bigcup_{n=1}^{\infty} X_{n}$, then $X$ is a weakly Stegall space.

Proof. Let $M$ be a complete metric space and let $\varphi: M \rightarrow 2^{X}$ be a minimal usco. For each $n \in \mathbb{N}$, let $M_{n}:=\left\{m \in M: \varphi(m) \cap X_{n} \neq \emptyset\right\}$. Then $\left\{M_{n}: n \in \mathbb{N}\right\}$ is a sequence of closed sets covering $M$, hence there is some $n_{0} \in \mathbb{N}$ such that $U:=\operatorname{int} M_{n_{0}} \neq \emptyset$. Now one can easily verify that the restriction of $\varphi$ to $U$ is a minimal usco which maps $U$ into $X_{n_{0}}$ (see, Lemma 3.1.2 of [1]). Therefore, since $X_{n}$ is a weakly Stegall space, there is some point $m \in U$ such that $\varphi(m)$ is a singleton.

Example 1. If Martin's axiom and the negation of the continuum hypothesis hold, then there exists a Banach space $X$ such that (i) $\left(X^{*}\right.$, weak $\left.{ }^{*}\right)$ is a weakly Stegall space and (ii) $\left(X^{*}\right.$, weak $\left.{ }^{*}\right)$ is not fragmentable.

Proof. In [4] the author gives an example, under the assumptions stated above, of a Banach space $X$ such that $\left(X^{*}\right.$, weak $\left.{ }^{*}\right)$ is not fragmentable but is in Stegall's class with respect to the class of all metrizable Baire spaces of weight at most $\aleph_{1}$. One can check that in this example the weight of $\left(B_{X^{*}}\right.$, weak $\left.{ }^{*}\right)$ is at most $\aleph_{1}$. Therefore, by Theorem $2\left(B_{X^{*}}\right.$, weak $\left.{ }^{*}\right)$ is a weakly Stegall space and so by Proposition $1,\left(X^{*}\right.$, weak $\left.^{*}\right)$ is a weakly Stegall space. 
Example 2. If there is a precipitous ideal on $\omega_{1}$ and Martin's axiom and the negation of the continuum hypothesis hold, then there exists a Banach space $X$ such that (i) $\left(X^{*}\right.$, weak $\left.{ }^{*}\right)$ is a weakly Stegall space and (ii) $\left(X^{*}\right.$, weak $\left.^{*}\right)$ is not a Stegall space.

Proof. In [4] the author shows that if there exists a precipitous ideal on $\omega_{1}$, then $\left(X^{*}\right.$, weak $\left.{ }^{*}\right)$ fails to be a Stegall space, where the Banach space $X$ is the same as in Example 1.

Remark 1. The author in [4 notes that the assumptions in Example 2 are equiconsistent with the existence of a measurable cardinal.

\section{A non-almost weak Asplund space}

In this section we give an example of a weakly Stegall compact space $K_{A}$ such that $\left(C\left(K_{A}\right),\|\cdot\|_{\infty}\right)$ is not an almost weak Asplund space. Let $B$ be a Bernstein subset of $(0,1)$, that is, $B$ is a subset of $(0,1)$ such that neither $B$ nor its complement contains any perfect compact sets (see [9, p. 23). We now set $A:=[(0,1 / 2) \cap B] \cup$ $[1 / 2+(0,1 / 2) \backslash B]$ (then $A$ does not contain any perfect compact subsets either), and define

$$
K_{A}:=[(0,1] \times\{0\}] \cup[(\{0\} \cup A) \times\{1\}] .
$$

We endow $K_{A}$ with the order topology generated by the lexicographical (i.e., dictionary) ordering (i.e., $\left(s_{1}, s_{2}\right) \leq\left(t_{1}, t_{2}\right)$ if, and only if, either $s_{1}<t_{1}$ or $s_{1}=t_{1}$ and $s_{2} \leq t_{2}$ ). It is shown in Proposition 2 of [3] that $K_{A}$ is a compact Hausdorff space.

Proposition 2 ([2, Proposition W3]). $K_{A}$ is a weakly Stegall space.

Proof. Let $M$ be a complete metric space, let $\varphi: M \rightarrow 2^{K_{A}}$ be a minimal usco and let $p: K_{A} \rightarrow[0,1]$ be defined by $p(t, \varepsilon):=t$ (i.e., $p$ is the natural projection). When $K_{A}$ is given the order topology and $[0,1]$ is given the usual topology, $p$ is continuous. Therefore by Lemma 1 (d) of [3], the composition mapping $p \circ \varphi: M \rightarrow 2^{[0,1]}$ is a minimal usco on $M$. Since $[0,1]$ is metrizable (and hence in Stegall's class) there exists a dense $G_{\delta}$ subset $G$ of $M$ such that $p \circ \varphi$ is single-valued at each point of $G$.

Now if $(p \circ \varphi)(m) \subseteq[0,1] \backslash A$ for some $m \in G$, then $\varphi$ is single-valued at $m$. Hence we need only consider the case when $(p \circ \varphi)(G) \subseteq A$. Since $A$ does not contain any perfect subsets, it follows from Proposition 7 (d) of [3] that there exists a non-empty open subset $U$ of $G$ and an element $a \in A$ such that $(p \circ \varphi)(U)=\{a\}$, i.e., $\varphi(U) \subseteq\{a\} \times\{0,1\}$. Now by applying Lemma 2 of [3] twice we see that the restriction of $\varphi$ to $U$ is still a minimal usco and since the two point set $\{a\} \times\{0,1\}$ is metrizable (and hence in Stegall's class) $\varphi$ must be single-valued at some points of $U$. This completes the proof.

We now show that $\left(C\left(K_{A}\right),\|\cdot\|_{\infty}\right)$ is not an almost weak Asplund space.

Lemma 1. Let $M: C\left(K_{A}\right) \rightarrow \mathbb{R}$ be defined by

$$
M(f):=\sup \left\{f(t, \varepsilon)+f\left(t+1 / 2, \varepsilon^{\prime}\right):\left(t, \varepsilon, \varepsilon^{\prime}\right) \in \Sigma\right\}
$$

where $\Sigma$ is given by

$\Sigma:=\left\{\left(t, \varepsilon, \varepsilon^{\prime}\right): 0 \leq t \leq 1 / 2\right.$ and $\varepsilon, \varepsilon^{\prime} \in\{0,1\}$ with $(t, \varepsilon) \in K_{A}$ and $\left.\left(t+1 / 2, \varepsilon^{\prime}\right) \in K_{A}\right\}$.

Then $M$ is a continuous convex function on $C\left(K_{A}\right)$. 
Proof. For each $\left(t, \varepsilon, \varepsilon^{\prime}\right) \in \Sigma$, define $x_{\left(t, \varepsilon, \varepsilon^{\prime}\right)}^{*}: C\left(K_{A}\right) \rightarrow \mathbb{R}$ by

$$
x_{\left(t, \varepsilon, \varepsilon^{\prime}\right)}^{*}(f):=f(t, \varepsilon)+f\left(t+1 / 2, \varepsilon^{\prime}\right) .
$$

Then each $x_{\left(t, \varepsilon, \varepsilon^{\prime}\right)}^{*}$ is a continuous linear functional on $C\left(K_{A}\right)$, (i.e., $x_{\left(t, \varepsilon, \varepsilon^{\prime}\right)}^{*} \in$ $\left.C\left(K_{A}\right)^{*}\right)$ and $\left\|x_{\left(t, \varepsilon, \varepsilon^{\prime}\right)}^{*}\right\|=2$. Now, $M(f)=\sup \left\{x_{\left(t, \varepsilon, \varepsilon^{\prime}\right)}^{*}(f):\left(t, \varepsilon, \varepsilon^{\prime}\right) \in \Sigma\right\}$. Therefore, as $M$ is the pointwise supremum of continuous (2-Lipschitz) linear functionals, $M$ is convex and 2-Lipschitz.

Lemma 2. $M(f)=\max \left\{f(t, \varepsilon)+f\left(t+1 / 2, \varepsilon^{\prime}\right):\left(t, \varepsilon, \varepsilon^{\prime}\right) \in \Sigma\right\}$ for each $f \in C\left(K_{A}\right)$.

Proof. We define $p: K_{A} \rightarrow[0,1]$ by $p(t, \varepsilon):=t$ (i.e., $p$ is the natural projection). When $K_{A}$ is given the order topology and $[0,1]$ is given the usual topology, $p$ is continuous. Hence $p^{-1}:[0,1] \rightarrow 2^{K_{A}}$ defined by $p^{-1}(t):=\left\{\left(t^{\prime}, \varepsilon\right): p\left(t^{\prime}, \varepsilon\right)=t\right\}$ is an usco mapping. So for each $f \in C\left(K_{A}\right)$ the mapping $T_{f}:[0,1 / 2] \rightarrow 2^{\mathbb{R}}$ defined by

$$
T_{f}(t):=f\left(p^{-1}(t)\right)+f\left(p^{-1}(t+1 / 2)\right)
$$

is an usco mapping, since sums and continuous images of usco mappings are again usco mappings. Thus, $T_{f}([0,1 / 2])$ is a compact subset of $\mathbb{R}$ and so,

$$
\begin{aligned}
M(f) & =\sup \left\{T_{f}(t): t \in[0,1 / 2]\right\} \\
& =\max \left\{T_{f}(t): t \in[0,1 / 2]\right\} \\
& =\max \left\{f(t, \varepsilon)+f\left(t+1 / 2, \varepsilon^{\prime}\right):\left(t, \varepsilon, \varepsilon^{\prime}\right) \in \Sigma\right\} .
\end{aligned}
$$

We will say that $\left(t, \varepsilon, \varepsilon^{\prime}\right) \in \Sigma$ supports $M$ at $f$ if $M(f)=f(t, \varepsilon)+f\left(t+1 / 2, \varepsilon^{\prime}\right)$. Let us now observe (using the notation of Lemma 1$)$ that if $\left(t, \varepsilon, \varepsilon^{\prime}\right)$ supports $M$ at $f$, then

$$
\begin{array}{r}
x_{\left(t, \varepsilon, \varepsilon^{\prime}\right)}^{*} \in \partial M(f):=\left\{x^{*} \in C\left(K_{A}\right)^{*}: x^{*}(g)-x^{*}(f) \leq M(g)-M(f)\right. \\
\text { for all } \left.g \in C\left(K_{A}\right)\right\} .
\end{array}
$$

Let us also recall that since $M$ is convex, $M$ is Gâteaux differentiable at $f$ if, and only if, $\partial M(f)$ is a singleton.

Lemma 3. Let $S: C\left(K_{A}\right) \rightarrow 2^{[0,1 / 2]}$ be defined by

$$
S(f):=\left\{t \in[0,1 / 2]:\left(t, \varepsilon, \varepsilon^{\prime}\right) \text { supports } M \text { at } f \text { for some } \varepsilon, \varepsilon^{\prime} \in\{0,1\}\right\} .
$$

Then $S$ is a minimal usco.

Proof. Let $f \in C\left(K_{A}\right)$ and let $T_{f}$ be defined as in Lemma 2, then $S(f)=\{t \in$ $\left.[0,1 / 2]: T_{f}(t) \cap[M(f), \infty) \neq \emptyset\right\}$. Therefore, $S(f)$ is a non-empty closed (and hence compact) subset of $[0,1 / 2]$. Consider $f \in C\left(K_{A}\right)$ and $U$ an open subset of $[0,1 / 2]$ containing $S(f)$. Let

$$
M_{[0,1 / 2] \backslash U}(f):=\max \left\{T_{f}(t): t \in[0,1 / 2] \backslash U\right\}<M(f) .
$$

Choose $0<\varepsilon<\left[M(f)-M_{[0,1 / 2] \backslash U}(f)\right] / 4$. Then an easy calculation shows that

$$
M_{[0,1 / 2] \backslash U}(g)<M(g)
$$

for all $g \in B(f ; \varepsilon)$ and so $S(g) \subseteq U$ for all $g \in B(f ; \varepsilon)$. This shows that $S$ is upper semi-continuous. The proof that $S$ is minimal is not difficult and is left to the reader. 
Example 3. The space $\left(C\left(K_{A}\right),\|\cdot\|_{\infty}\right)$ is not almost weak Asplund.

Proof. We show that $C\left(K_{A}\right)$ is not an almost weak Asplund space by showing that the continuous convex function $M$ defined on $C\left(K_{A}\right)$ is non-Gâteaux differentiable at the points of a residual subset of $C\left(K_{A}\right)$. Let $S: C\left(K_{A}\right) \rightarrow 2^{[0,1 / 2]}$ be defined as in Lemma 3 and let $G:=\left\{f \in C\left(K_{A}\right): S(f)\right.$ is a singleton $\}$. By Lemma 3 and the fact that $[0,1 / 2]$ is metrizable and hence in Stegall's class, $G$ is a residual (and hence dense) subset of $C\left(K_{A}\right)$. As in Lemma 2, let $p: K_{A} \rightarrow[0,1]$ denote the natural projection of $K_{A}$ onto $[0,1]$ and for each $n \in \mathbb{N}$ let,

$$
\begin{aligned}
O_{1 / n}:=\left\{f \in C\left(K_{A}\right): \operatorname{diam}\left[f\left(p^{-1}(S(f))\right)\right]\right. & <1 / n \text { and } \\
& \left.\operatorname{diam}\left[f\left(p^{-1}(S(f)+1 / 2)\right)\right]<1 / n\right\} .
\end{aligned}
$$

Since both mappings $f \mapsto f\left(p^{-1}(S(f))\right)$ and $\left.f \mapsto f\left(p^{-1}(S(f)+1 / 2)\right)\right)$ are usco mappings, it is easy to see that each $O_{1 / n}$ is an open subset of $C\left(K_{A}\right)$. We claim that each $O_{1 / n}$ is dense in $C\left(K_{A}\right)$. To justify this assertion let us fix $n \in \mathbb{N}, f_{0} \in G$ and $\varepsilon>0$, with the goal of showing that $O_{1 / n} \cap B\left(f_{0}, \varepsilon\right) \neq \emptyset$. We shall only consider the case when $S\left(f_{0}\right):=\left\{t_{0}\right\} \subseteq[0,1 / 2) \backslash B$ as the case when $S\left(f_{0}\right) \subseteq B$ is similar. By the continuity of $f_{0}$ there exists a $\delta>0$ such that

(i) $\left|f_{0}(t, \varepsilon)-f_{0}\left(t_{0}, 0\right)\right|<1 / 4 n$ for all $(t, \varepsilon) \in K_{A}$ with $\left|t-t_{0}\right|<\delta$;

(ii) $\left|f_{0}(t+1 / 2, \varepsilon)-f_{0}\left(t_{0}+1 / 2,1\right)\right|<1 / 4 n$ for all $(t+1 / 2, \varepsilon) \in K_{A}$ with $t \in$ $\left(t_{0}, t_{0}+\delta\right)$

(iii) $\left|f_{0}(t+1 / 2, \varepsilon)-f_{0}\left(t_{0}+1 / 2,0\right)\right|<1 / 4 n$ for all $(t+1 / 2, \varepsilon) \in K_{A}$ with $t \in$ $\left(t_{0}-\delta, t_{0}\right)$.

Since the mapping $S$ is upper semi-continuous there exists a $0<\delta^{\prime}<1 / 4 n$ such that $S\left(B\left(f_{0} ; \delta\right)\right) \subseteq\left(t_{0}-\delta, t_{0}+\delta\right)$. Now, if $\left|f_{0}\left(t_{0}+1 / 2,0\right)-f_{0}\left(t_{0}+1 / 2,1\right)\right|<1 / n$, then $O_{1 / n} \cap B\left(f_{0} ; \varepsilon\right) \neq \emptyset$. So we will assume that $\left|f_{0}\left(t_{0}+1 / 2,0\right)-f_{0}\left(t_{0}+1 / 2,1\right)\right| \geq 1 / n$. In fact, we will assume that $f_{0}\left(t_{0}+1 / 2,1\right)-f_{0}\left(t_{0}+1 / 2,0\right) \geq 1 / n$. By judiciously choosing a "bump function" $h: K_{A} \rightarrow[0, \infty)$ with $\|h\|_{\infty}<\delta^{\prime}$ and

$$
\operatorname{supp}(h):=\{(t, \varepsilon): h(t, \varepsilon) \neq \emptyset\} \subseteq p^{-1}\left(\left(t_{0}+1 / 2, t_{0}+1 / 2+\delta\right)\right)
$$

we can assume that $M\left(f_{0}\right)<M\left(f_{0}+h\right)$. In fact, with this choice of function we can show that $S\left(f_{0}+h\right) \subseteq\left(t_{0}, t_{0}+\delta\right)$. Indeed, if $t \in S\left(f_{0}+h\right)$ and $t \leq t_{0}$, then

$$
\begin{aligned}
& \left(f_{0}+h\right)(t, \varepsilon)+\left(f_{0}+h\right)\left(t+1 / 2, \varepsilon^{\prime}\right) \\
& \quad=f_{0}(t, \varepsilon)+f_{0}\left(t+1 / 2, \varepsilon^{\prime}\right) \leq M\left(f_{0}\right)<M\left(f_{0}+h\right)
\end{aligned}
$$

which contradicts the fact that $t \in S\left(f_{0}+h\right)$. It is now a straightforward calculation to show that

$$
\begin{aligned}
\operatorname{diam}\left[\left(f_{0}+h\right)\left(p^{-1}\left(S\left(f_{0}+h\right)\right)\right)\right] & <1 / n \text { and } \\
& \operatorname{diam}\left[\left(f_{0}+h\right)\left(p^{-1}\left(S\left(f_{0}+h\right)+1 / 2\right)\right)\right]<1 / n .
\end{aligned}
$$

Therefore $\left(f_{0}+h\right) \in O_{1 / n}$ and so $B\left(f_{0} ; \varepsilon\right) \cap O_{1 / n} \neq \emptyset$. We now claim that if $f \in \bigcap_{n=1}^{\infty} O_{1 / n}$, then $M$ is not Gâteaux differentiable at $f$. To see this consider the sets

$$
\Sigma(t):=\left\{\left(t^{\prime}, \varepsilon, \varepsilon^{\prime}\right) \in \Sigma: t^{\prime}=t\right\} \quad \text { for all } t \in[0,1 / 2] .
$$

Now $|\Sigma(t)|=2$ for each $t \in[0,1 / 2)$ and if $t \in S(f)$, then each member of $\Sigma(t)$ supports $M$ at $f$, i.e., for each $\left(t, \varepsilon, \varepsilon^{\prime}\right) \in \Sigma(t), x_{\left(t, \varepsilon, \varepsilon^{\prime}\right)}^{*}$ is a subgradient of $M$ at $f$. Therefore $M$ is not Gâteaux differentiable at $f$. This completes the proof. 
More information on both weakly Stegall and almost weak Asplund spaces will be contained in [10].

\section{REFERENCES}

1. Marián J. Fabian, Gâteaux differentiability of convex functions and topology. Weak Asplund spaces, Canadian Mathematical Society Series of Monographs and Advanced Texts, WileyInterscience, New York, 1997. MR 98h:46009

2. Ondřej F. K. Kalenda, Weak Stegall spaces, unpublished manuscript, Spring 1997 (3 pages).

3. Ondřej F. K. Kalenda, Stegall compact spaces which are not fragmentable, Topology Appl. 96 (1999), 121-132. MR 2000i:54027

4. Ondřej F. K. Kalenda, A weak Asplund space whose dual is not in Stegall's class, Proc. Amer. Math. Soc. 130 (2002), 2139-2143.

5. Petar S. Kenderov, Ivaylo S. Kortezov and Warren B. Moors, Continuity points of quasicontinuous mappings, Topology Appl. 109 (2001), 321-346. MR 2001m:54008

6. Petar S. Kenderov and Warren B. Moors, Game characterization of fragmentability of topological spaces, Mathematics and Education in Mathematics 8-18, Proceedings of the 25th Spring Conference of the Union of Bulgarian Mathematicians, April 1996, Kazanlak, Bulgaria (1996).

7. Petar S. Kenderov and Warren B. Moors, Fragmentability and sigma-fragmentability of Banach spaces, J. London Math Soc. 60 (1999), 203-223. MR 2001f:46025

8. Petar S. Kenderov, Warren B. Moors and Scott Sciffer, A weak Asplund space whose dual is not weak* fragmentable, Proc. Amer. Math. Soc. 129 (2001), 3741-3747.

9. John C. Oxtoby, Measure and category. A survey of the analogies between topological and measure spaces, Graduate Texts in Mathematics, Vol. 2. Springer-Verlag, New York, 1971. MR 52:14213

10. Sivajah Somasundaram, Almost weak Asplund spaces, $\mathrm{PhD}$ Thesis, The University of Waikato, Hamilton, New Zealand (in preparation).

Department of Mathematics, The University of Waikato, Private Bag 3105, Hamilton 2001, New ZEALAND

E-mail address: moors@math.waikato.ac.nz

Department of Mathematics, The University of Waikato, Private Bag 3105, Hamilton 2001, NeW ZEALAND

E-mail address: ss15@math.waikato.ac.nz 